\title{
Do effective properties for unsaturated weakly layered porous media exist? An experimental study
}

\author{
A. Bayer ${ }^{1}$, H.-J. Vogel ${ }^{1}$, O. Ippisch ${ }^{2}$, and K. Roth $^{1}$ \\ ${ }^{1}$ Institute of Environmental Physics, University of Heidelberg, Im Neuenheimer Feld 229, 69120 Heidelberg, Germany \\ ${ }^{2}$ Interdisciplinary Center for Scientific Computing, University of Heidelberg, Im Neuenheimer Feld 368, 69120 Heidelberg, \\ Germany
}

Received: 12 May 2005 - Published in Hydrology and Earth System Sciences Discussions: 21 June 2005

Revised: 20 September 2005 - Accepted: 11 October 2005 - Published: 31 October 2005

\begin{abstract}
In a multi-step outflow experiment we found that a weak heterogeneity within a sand column prevents the estimated effective hydraulic parameters from being unique. We compared vertical water content profiles calculated from these parameters with profiles measured by $\mathrm{x}$-ray attenuation. A layered material model based on $\mathrm{x}$-ray data was able to reproduce the outflow curve and also the water content distribution inside the column. We also calculated effective parameters for the layered model turned upside down and obtained large differences to the set of values of the original sample.
\end{abstract}

\section{Introduction}

Formulating the dynamics of soil water movement at some continuum scale leads to the introduction of effective material properties, most importantly the soil water characteristic $\theta\left(\psi_{m}\right)$ and the tensorial function $\mathrm{K}(\theta)$ of the hydraulic conductivity. These equilibrium relations represent the complicated sub-scale structures and processes, i.e. those at scales below the one at which the dynamics is formulated, all the way down to the pore scale. A broad and continuing effort aims at understanding and eventually predicting effective material properties based on simplified representations of sub-scale structures and processes, (e.g. Arya and Paris, 1981; Rajarama et al., 1997; Vogel, 2000). Except for a few simple materials, this aim has not yet been reached, however. Consequently, effective properties are generally determined experimentally. Typically, undisturbed samples with a characteristic length of some $0.1 \mathrm{~m}$ are extracted from the site of interest, perturbation-relaxation experiments are performed, for example of the multi-step outflow type (van Dam et al., 1994), and the desired effective properties are obtained by

Correspondence to: A. Bayer

(andreas.bayer@iup.uni-heidelberg.de) inverse modelling based on Richards' equation and the measured outflow. These properties are subsequently used for numerical simulations of the water flow at the site of interest. The question is: are effective hydraulic properties defined for unsaturated flow unique, in the sense that they are applicable for all flow regimes, not just for the one for which they were estimated. This would in particular imply that turning the column upside down and repeating the relaxation experiment yields the same parameters as the experiment in the original orientation. This requirement is obvious for $\theta\left(\psi_{m}\right)$ which is a scalar quantity but can also be shown to hold for the tensorial quantity $\mathrm{K}(\theta)$. While this is trivially satisfied in hypothetical uniform media, we demonstrate in the following that already in a weakly heterogeneous layered sand column, effective hydraulic properties depend on the flow direction and are thus not unique. As a consequence of this, we may expect that the effective properties obtained from a multi-step outflow experiment will lead to a correct simulation of the drainage of a soil profile. However, already infiltration but certainly evaporation may not be described correctly. This has obvious implication for the simulation of soil-atmosphere coupling where the direction of the flow changes regularly.

\section{Material and methods}

We determined the effective material properties $\theta\left(\psi_{m}\right)$ and $\mathrm{K}(\theta)$ in a standard multi-step outflow (MSO) experiment (van Dam et al., 1994). Sand with $0.25-0.63 \mathrm{~mm}$ grain size was filled into a PVC column with $10 \mathrm{~cm}$ height and a radius of $8.15 \mathrm{~cm}$. The column was mounted on a porous plate with an air-entry pressure of $250 \mathrm{hPa}$. The water potential at the bottom of the sample was controlled and the water outflow was collected in a burette and its amount monitored during the experiment using a pressure sensor. The upper end of the sample was not sealed, but covered with a PVC-plate to

(C) 2005 Author(s). This work is licensed under a Creative Commons License. 


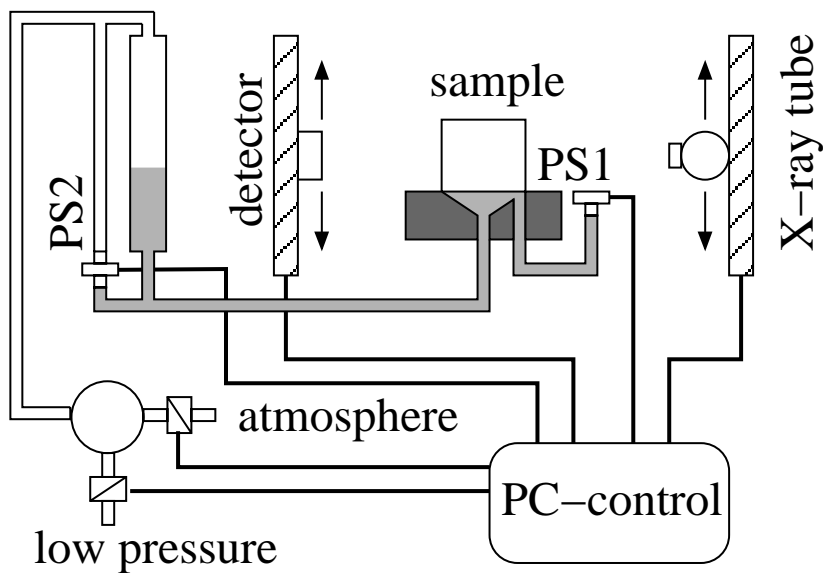

Fig. 1. Sketch of the experimental setup: The sample is placed between the $\mathrm{x}$-ray source and the line detector, which both can be moved vertically. The pressure at the lower boundary is measured by the sensor PS 1 and controlled by a computer. The water outflow is collected in a burette and measured using the pressure sensor PS2.

minimize evaporation. The column was placed between a $\mathrm{x}$-ray source and a horizontal line sensor. This configuration allows us to monitor the water content during the MSO experiment by recording $\mathrm{x}$-ray attenuation profiles. All operations are electronically controlled by a computer. A sketch of the setup is shown in Fig. 1.

The $\mathrm{x}$-ray system consists of a polychromatic medical $\mathrm{x}$-ray source operated at $141 \mathrm{kV}$ acceleration voltage and $20 \mathrm{~mA}$ current, together with a 12 bit CCD line sensor including 1280 quadratic pixels of $0.4 \mathrm{~mm}$ side length. Tube and detector were mounted $1.32 \mathrm{~m}$ apart and could be moved synchronously in vertical direction. The sample was placed in a distance of $0.55 \mathrm{~m}$ from the tube and scanned with a vertical resolution of $0.4 \mathrm{~mm}$ during $35 \mathrm{~s}$. In front of the tube we used a bow-tie filter made of PVC to homogenize the transmitted intensities on the detector with respect to the cylindrical sample geometry.

The initial condition for the MSO experiment was complete water saturation, i.e. the matric head at the lower boundary was $h_{\mathrm{LB}}=\frac{\psi_{m}}{\rho_{w} g}=10 \mathrm{~cm}$, where $\psi_{m}$ is matric potential, $\rho_{w}$ the mass density of water and $g$ the acceleration of gravity. To ensure full water saturation the sand was filled into the rising water table. During the filling procedure, the water table was adjusted in a way that it was always below but close to the sand surface. With this method the amount of air entrapments during saturating the sample can be minimized and the arrangement of sand grains is stabilized due to capillary forces.

During the MSO experiment the sample was desaturated in eight steps (Fig. 2) from $h_{L B}=10 \mathrm{~cm}$ to $-20 \mathrm{~cm}$. At different pressure steps $(10,5,2,0,-4,-8,-12,-16$ and $-20 \mathrm{~cm}$ ) vertical $\mathrm{x}$-ray attenuation profiles of the sam-

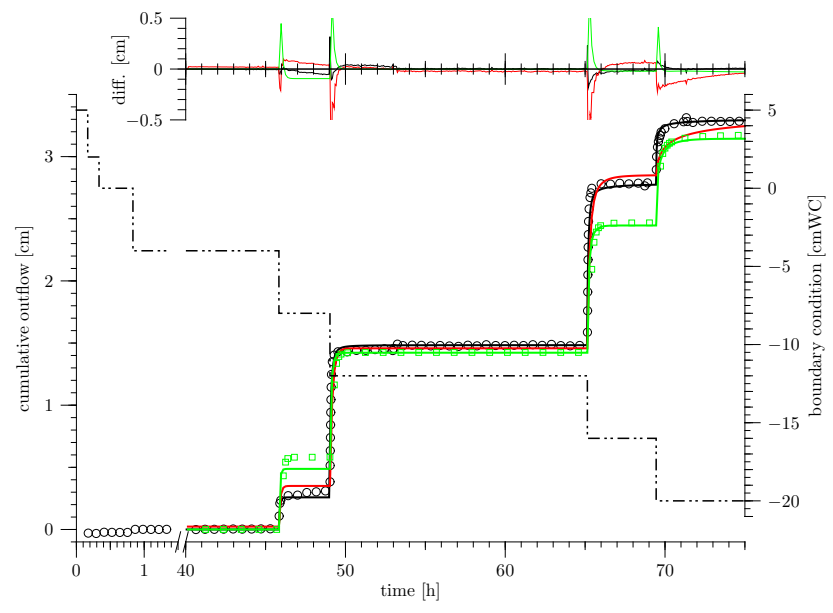

Fig. 2. The circles show the measured cumulative outflow during a MSO experiment. The result of inverse modeling is shown as black line and the forward simulated outflow for a layered material model is represented by the red line. Green squares show the simulated outflow with the structured material model turned upside down and the result of inverse modeling of this data is plotted as a green line. Pressure at the lower boundary is plotted as dash-dot-dot-line and related to the right y-axis. Differences between measurement and modeling are shown in the upper part with corresponding colors. See Figs. 3 and 5 to appreciate why the red curve is deemed to belong to a better representation than the black one.

ple were recorded. This was done $1 \mathrm{~h}$ to $3 \mathrm{~h}$ after the pressure step was established to ensure that the system had approached hydrostatic equilibrium. From the measured $\mathrm{x}$-ray intensities $I(x, z)$ and $I_{0}(x)$, with and without the sample respectively, $\mathrm{x}$-ray attenuation $A(x, z)$ is calculated as

$A(x, z)=\ln \left(\frac{I_{0}(x)}{I(x, z)}\right)$,

where $x$ denotes the pixel position on the detector and $z$ its vertical position. For each depth $z$ the $A(x, z)$ was measured across the center of the cylindrical sample including 30 pixel on the detector line which corresponds to a width of $1.2 \mathrm{~cm}$. In this range the path length of the beam through the material is nearly constant and the signal to noise ratio is improved by averaging.

The effective attenuation can be decomposed into the contributions of the different constituents weighted by their volume fraction leading to

$A(z)=(1-\phi) \mu_{\mathrm{m}} d+\theta \mu_{\mathrm{w}} d+O$,

where $\mu_{\mathrm{m}}$ and $\mu_{\mathrm{w}}$ are the linear attenuation coefficients of matrix and water, respectively. The diameter of the sample is $d, \phi$ is the porosity of the material and $\theta$ the volumetric water content that we are interested in. The attenuation of the other contributed materials, like air and surrounding PVC cylinder, is represented by $O$. X-ray attenuation of air is by two orders of magnitude lower than that of the other materials (Hubbel, 
1982) and hence, it was neglected for further calculations. We also neglected beam harding effects due to the polychromatic spectra of the $\mathrm{x}$-ray source and the energy dependence of attenuation. This can be done as the path length of the $\mathrm{x}$-ray beam through the column was almost constant and the material didn't change on this way.

However, the resulting non-linear attenuation along that path has to be considered when calculating the absolute volume fraction of the different constituents. As described below, this was done by introducing a correction factor which can be obtained by calibration. We assumed that the water content $\theta$ is the only changing quantity during the experiment and that the porosity $\phi(z)$ is equal to the saturated water content $\theta_{s}(z)$ for each depth $z$. Then, the water content profile $\theta(z)$ can be obtained from the difference between $A\left(\theta_{s}(z)\right)$ and $A(\theta(z))$ through

$\theta(z)=\theta_{s}-\beta \frac{A\left(\theta_{s}(z)\right)-A(\theta(z))}{\mu_{\mathrm{w}} d}$.

We used $\mu_{\mathrm{w}}=0.0188 \mathrm{~mm}^{-1}$. The unknown correction factor $\beta$ which accounts for the non-linear attenuation, was determined from the measured cumulative water outflow during the experiment. For different pressure steps $p_{i}$, the mean water content $\hat{\theta}\left(p_{i}\right)$ can be obtained by

$\hat{\theta}\left(p_{i}\right)=\theta_{s}-\frac{q\left(p_{i}\right)}{V}$,

where $q\left(p_{i}\right)$ is the measured volume of emanated water and $V$ is the volume of the sample. The amount of outflow between two pressure steps $p_{i}$ and $p_{j}$ can be calculated from Eq. (4) by

$\Delta \hat{\theta}\left(p_{i}, p_{j}\right)=\theta_{s}-\frac{q\left(p_{i}\right)-q\left(p_{j}\right)}{V}$.

Clearly, this calculation is only allowed for states of hydrostatic equilibrium for a given pressure step $p_{i}$. Then, the relation

$\hat{\theta}\left(p_{i}\right)=\int_{0}^{z_{\max }} \theta\left(z, p_{i}\right) \mathrm{d} z$

should be satisfied for $\hat{\theta}\left(p_{i+1}\right)-\hat{\theta}\left(p_{i}\right)$. Using Eq. (3) to calculate $\theta\left(z, p_{i}\right)$ we chose the correction factor $\beta$ such that relation (Eq. 6) is fulfilled. This could be done for different pressure steps which led to a value of $\beta=2.17 \pm 0.13$. We used the pressure steps between $h_{L B}=-4 \mathrm{~cm}$ and $-20 \mathrm{~cm}$, because during the previous steps close to water saturation we observed some changes in the structure of the upper few millimeters of the sample which would introduce an error. But for the latter steps the matrix is stabilized and the difference between two following profiles is only affected by the outflow of water. The relative large value of $\beta$ results from the different contributions of sand and water to the measured attenuation. The attenuation of sand is about four times larger than that of water with respect to volume fraction. This leads to apparent changes in water content originating from non-linear attenuation.
Table 1. Column $A \uparrow$ contains best-fit values determined by inverse modeling of the measured outflow curve. Col. $B \downarrow$ shows the results of fitting the simulated outflow data for the sample turned upside down. Parameters with empty boxes in col. $B \downarrow$ were set to the corresponding value in col. $A \uparrow$ and held constant. The sum of the squared differences between the original and fitted curve are in row $S S Q$. Uncertainties are calculated using the $\chi 2$ statistics as described by Press et al. (1992).

\begin{tabular}{ccc}
\hline parameter & $A \uparrow$ & $B \downarrow$ \\
\hline$K_{S}(\mathrm{~cm} / \mathrm{h})$ & $84.9 \pm 9.5$ & \\
$n(-)$ & $24.1 \pm 1.2$ & $6.31 \pm 0.14$ \\
$\alpha\left(10^{-2} \mathrm{~cm}^{-1}\right)$ & $-5.73 \pm 0.01$ & $-5.68 \pm 0.03$ \\
$\theta_{r}$ & $0.060 \pm 0.001$ & 0.0 \\
& & \\
$\theta_{S}$ & $0.393 \pm 0.008$ & \\
$\tau$ & 0.5 & 1.66 \\
$S S Q$ & 0.68 & \\
\hline
\end{tabular}

\section{Results and discussion}

The resulting outflow curve of the MSO experiment is shown in Fig. 2 together with the lower boundary condition.

The time axis is split into two pieces: the first part shows the result for pressure $p \geq 0 \mathrm{~cm}$ where nearly no outflow was recorded as the air entry value was not yet reached. This part was excluded from further evaluation. The outflow data were set to zero at $t=40 \mathrm{~h}$, which was the starting point for inverse modeling. Hydraulic parameters were estimated by solving the inverse problem of Richards' equation together with a Mualem/van Genuchten (Mualem, 1976; van Genuchten, 1980) parameterization of the soil-water retention and hydraulic conductivity curve. A one-dimensional, fully-implicit, finite differences mixed formulation (Celia et al., 1990) was used together with the Levenberg-Marquardt algorithm, to optimize the parameters (Zurmühl, 1998). This approach assumes the sample to be a homogenous effective medium. The resulting, optimized parameters are shown in Table 1, the related best-fit curve in Fig. 2. The saturated volumetric water content $\theta_{s}$ was assumed to be equal to porosity $\phi=0.393 \pm 0.008$ calculated from the mass of sand, its density and the volume of the sample.

The modeled outflow curve fits very well to the measured data and uncertainties of the fitted parameters are small indicating that the values represent the hydraulic properties of the sample in an acceptable way.

Based on the optimized parameters the vertical distribution of water was calculated to compare it with the directly measured $\mathrm{x}$-ray attenuation profiles. This was done for hydrostatic states where $\mathrm{x}$-ray attenuation profiles were taken. The measured and calculated profiles are shown in Fig. 3. 


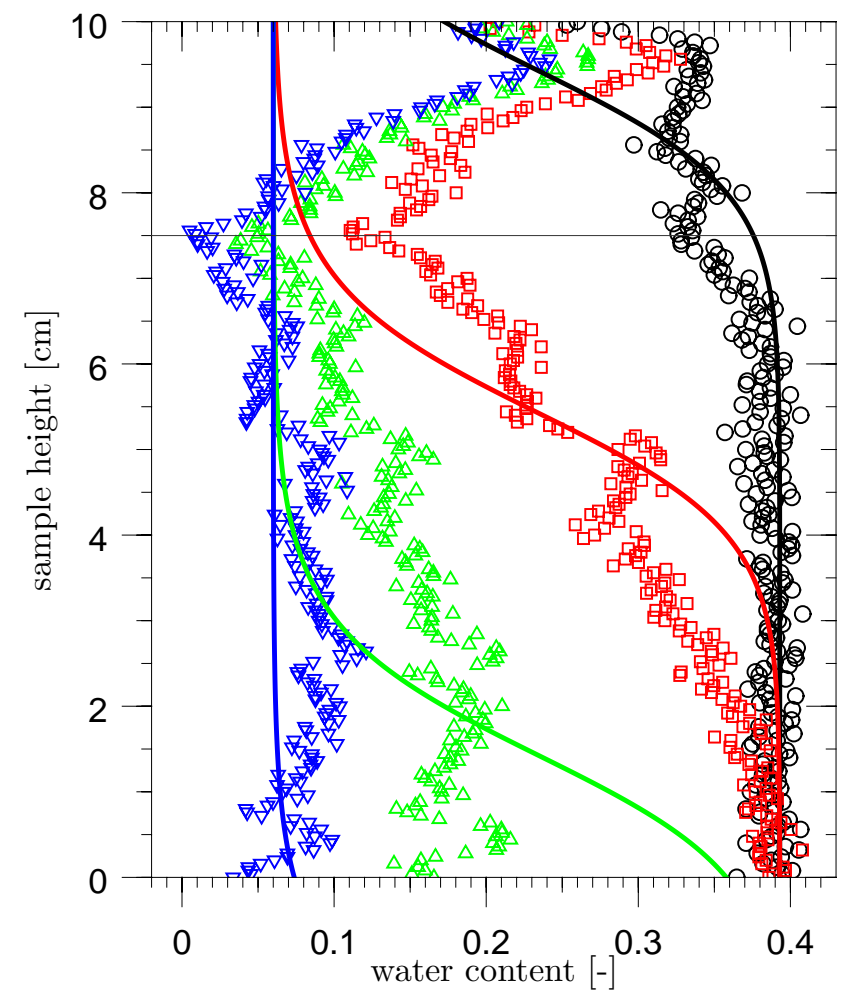

Fig. 3. Measured (symbols) and simulated (solid lines) vertical water content profiles assuming a homogenous sample. The matric head at the lower boundary was $-8 \mathrm{~cm}$ (black), $-12 \mathrm{~cm}$ (red), $-16 \mathrm{~cm}$ (green) and $-20 \mathrm{~cm}$ (blue) for the shown profiles. The hydraulic parameters were estimated by inverse modeling of the MSO experiment. Above the horizontal line $(z=7.5 \mathrm{~cm})$ the measurements are affected by changes near the sample surface that leads to unrealistic water content values.

Obviously, the parameters estimated from outflow data cannot reflect the internal behavior of the sample. Especially the profile at $h_{L B}=-16 \mathrm{~cm}$ shows large differences between measurement and prediction. The top part of the sample is affected by a slightly increasing density of the material during the experiment that lead to large changes in the measured attenuation because of the large attenuation coefficient for sand compared to that of water. If the sand surface sinks by $1 \mathrm{~mm}$ due to compaction of the upper $2 \mathrm{~cm}$ of the sample the attenuation leads to an overestimation of the water content by 0.2 .

The attenuation data clearly show that the structure is not homogenous in the vertical direction, as the shape of the different profiles is not similar. To include heterogeneity we generated a material model based on the measured $\mathrm{x}$-ray attenuation profiles. Since attenuation data were measured in hydrostatic equilibrium we can extract some points on the water retention curve at different vertical positions of the sample. The matric head $h[\mathrm{cmWC}$ ) for any point on the vertical z-axis is given by $h(z)=h(0)-z$, where $h(0)$ is the matric head at the lower boundary of the sam-
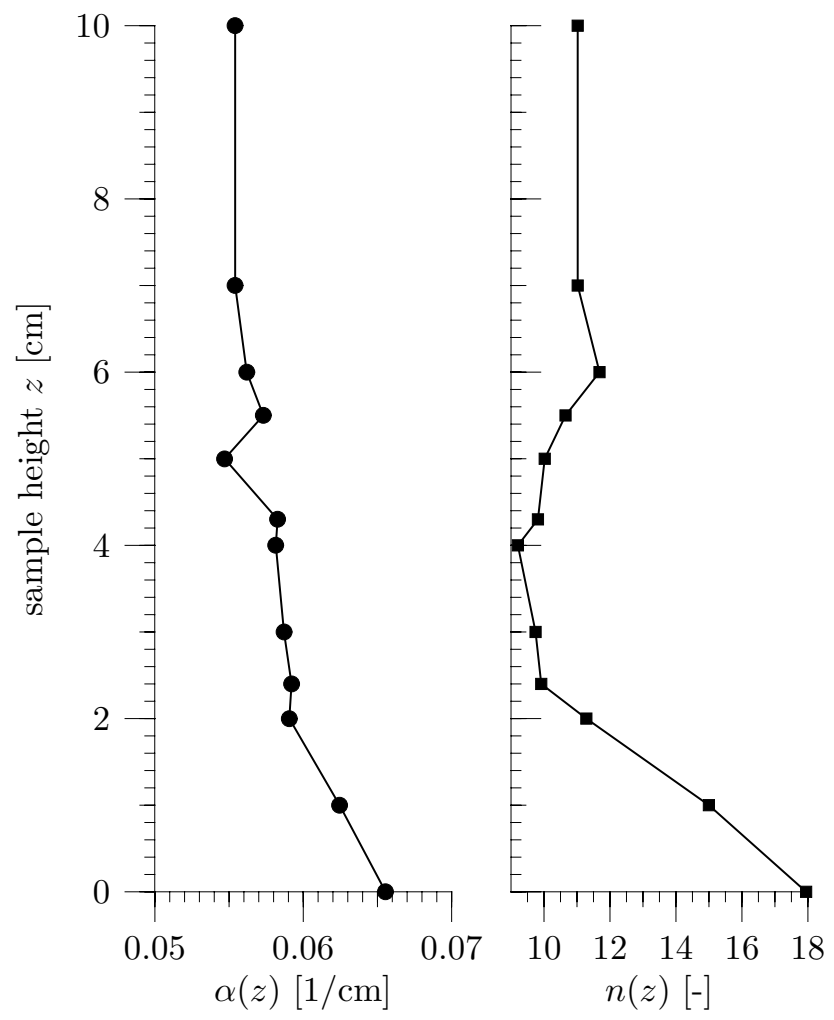

Fig. 4. Fitted van Genuchten parameters $\alpha(z)$ and $n(z)$.

ple and $z$ is the height above it. Corresponding to the eight steps of the MSO experiment we obtained eight points on the water retention curves $\theta_{z}(h)$. For a discrete material model of our sample we used the attenuation data at $z=0 \mathrm{~cm}, \ldots, 7 \mathrm{~cm}$ in steps of $\Delta z=1 \mathrm{~cm}$ and three additional ones at $z=2.4 \mathrm{~cm}, 4.3 \mathrm{~cm}, 5.5 \mathrm{~cm}$ to account for the structure indicated by the non-monotonic nature of the measured water content profiles (Fig. 5). A van Genuchten parameterization was fitted to the extracted points $\theta_{z}(h)$ to obtain a set of parameters $n(z)$ and $\alpha(z)$ (Fig. 4). These values were used to create a layered material model with respect to van Genuchten parameters. Values between the supporting points at different heights where found by linear interpolation. Parameters for $z>7 \mathrm{~cm}$ were set to the values at $z=7 \mathrm{~cm}$ because of the uncertainty of the values in this region. The saturated hydraulic conductivity was set proportional to the square of $\alpha(z) / \alpha^{*}$, in analogy to Miller media (Miller and Miller, 1956), where $\alpha^{*}$ is the value found in the MSO data. The harmonic average of the hydraulic conductivities of each layer weighted by its thickness leads to an effective saturated hydraulic conductivity for the layered material model of $85.7 \mathrm{~cm} / \mathrm{h}$ which is very close to the value of the unstructured model (see Table 1). The values for saturated water content $\theta_{s}$ and for the tortuosity parameter $\tau$ were set to 0.393 and 0.5 , respectively. We simulated the MSO experiment for the heterogeneous structure numerically by solving 
Richards' equation by a cell-centered finite-volume scheme with full-upwinding in space and an implicit Euler scheme in time. Linearization of the nonlinear equations is done by an inexact Newton-Method with line search. The linear equations are solved with an algebraic multigrid solver. For the time solver the time step is adopted automatically. The result shows a much better agreement of measured and simulated water content profiles (Fig. 5).

The main features of the measured profiles are reproduced very well. Also the outflow is modeled reasonably well, except for the last pressure step. The hydraulic conductivity seems to be underestimated by the model resulting in a much slower convergence to hydrostatic equilibrium compared to the experiment. This is a consequence of the perfect layering of the modeled structure with the coarsest textured material at the bottom (parameter $\alpha(z)$, Fig. 4). With decreasing water content the conductivity of this layer is reduced first and hinders the water outflow from above. In reality, this layering is expected to be less perfect which would explain the discrepancy between model and experiment.

Presumably, the layered heterogeneity of our sample was generated during sample preparation, when the sand was poured into the rising water table. At the beginning of the filling procedure the water table was at $z \approx 1 \mathrm{~cm}$. Afterwards the water table was near or below the sand surface. This leads to increasing capillary forces during the filling procedure and thus to an increasing compaction of the sand. This is reflected by the parameter $\alpha(z)$ which decreases with sample height. In our experiment, the weak, layered heterogeneity was created unintentionally when filling the column. However, in natural soils, similar heterogeneities are expected to be the rule rather than the exception. Hence, the question arises: What are the implications when presuming a weakly layered heterogeneous sample to be homogeneous for the purpose of parameter estimation? Notice that this is done tacitly in all current procedures for measuring flow and transport parameters.

Remarkably, in our experiment, the estimation of effective parameters assuming a homogeneous material is not at all affected by the present heterogeneity since the inversion of Richards' equation yields an almost ideal description of the measured cumulative outflow curve (Fig. 2). This questions the significance and uniqueness of the obtained parameters which should not depend on details of the measurement procedure and on the specific flow regime.

For a horizontally layered material it has to be expected that its hydraulic behavior depends on the orientation of the investigated sample. This is especially true for materials having a highly non-linear water retention curve $\theta(h)$ with changing water content along the vertical axis of a sample at hydrostatic equilibrium. In this case the hydraulic behavior of the different layers depends on their vertical position within the sample. Consequently the effective hydraulic parameters obtained from MSO experiments are expected to depend on the orientation of the sample.

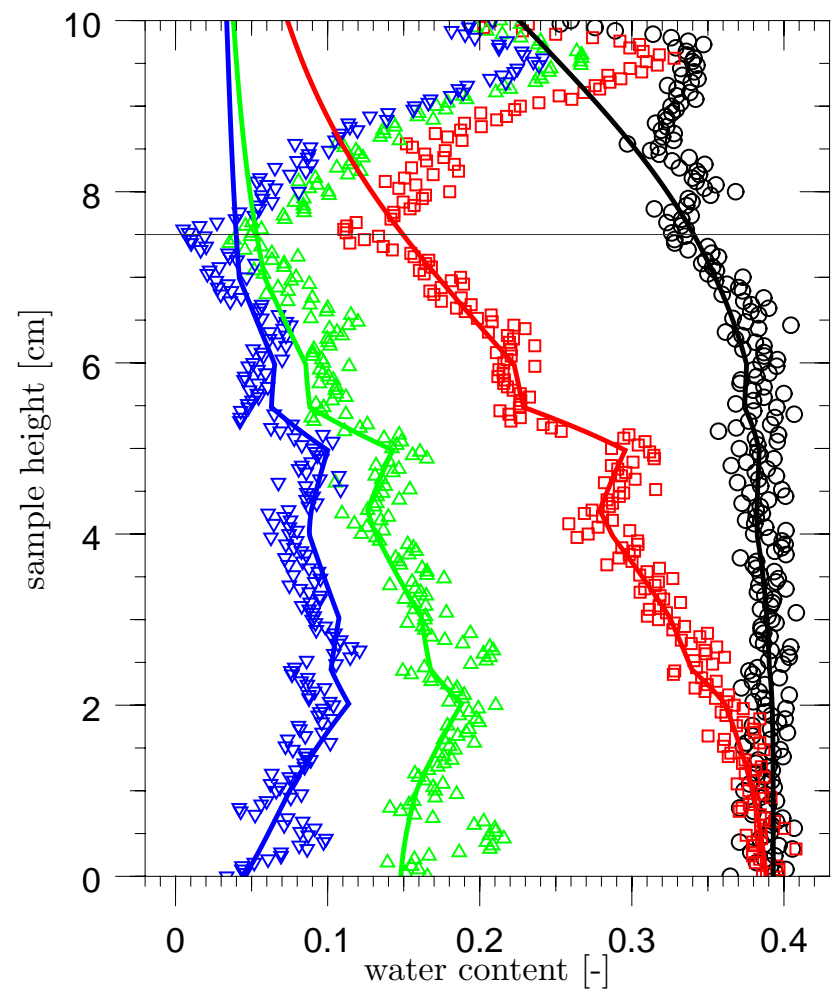

Fig. 5. Measured (symbols) and simulated (solid lines) vertical water content profiles assuming a layered sample. The matric head at the lower boundary was $-8 \mathrm{~cm}$ (black), $-12 \mathrm{~cm}$ (red), $-16 \mathrm{~cm}$ (green) and $-20 \mathrm{~cm}$ (blue) for the shown profiles. Above the horizontal line $(z=7.5 \mathrm{~cm})$ the measurements are affected by changes near the sample surface that leads to unrealistic water content values.

For our sample, this could be checked by simulating a MSO experiment with a reversed orientation of the sample. Thus the less compacted part was on top and the more compacted at the bottom. For technical reasons our setup does not allow the undisturbed rotation of a sample, and corresponding experiment was not possible. As shown in Fig. 2, the simulated outflow curve for the reversed sample orientation differs considerably from the original one. In contrast to the original experiment, the coarse textured layers on top of the sample drain earlier during the MSO experiment and the fine textured layers at the bottom retain more water which leads to a reduction of the cumulative outflow at the end of the experiment.

The inverse estimation of effective parameters assuming a homogeneous material was again successful, i.e. Richards' equation could describe the cumulative outflow reasonably well (Fig. 2) resulting in a different but unique set of hydraulic parameters (Table 1). Only $n, \alpha$ and $\theta_{r}$ were fitted, the other parameters were set to the values found in the first inversion. The resulting pressure-saturation relations and the hydraulic conductivity curves are shown in Fig. 6. 


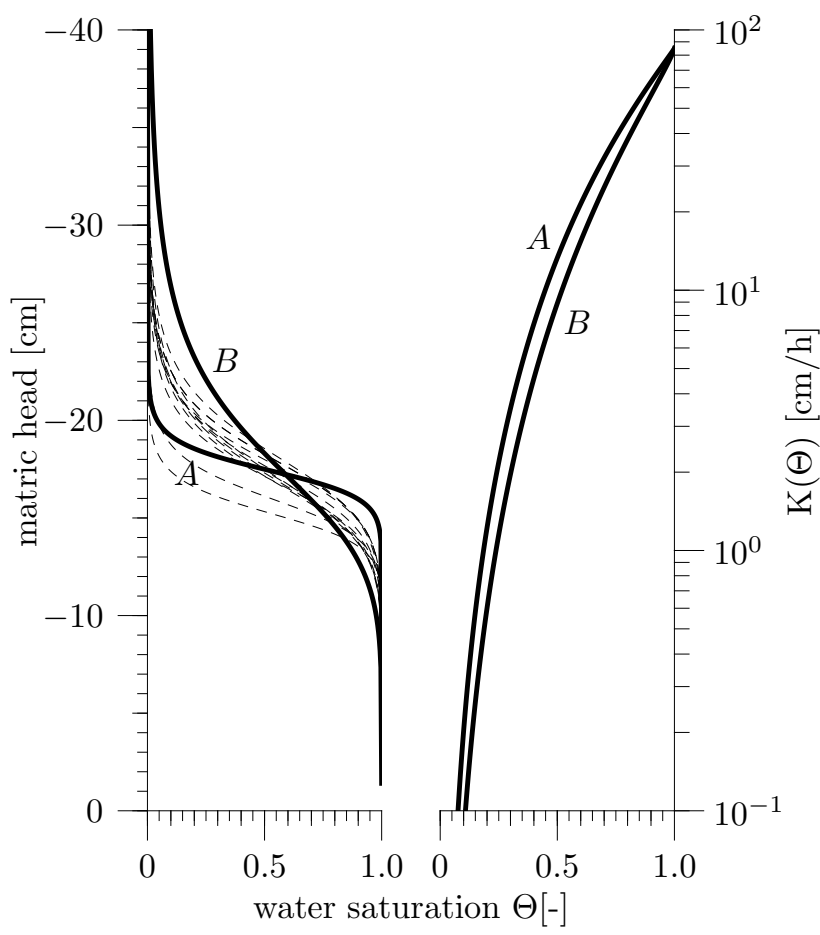

Fig. 6. Water retention curve (left) and hydraulic conductivity (right) for the two flow directions. Parameters for the respective curve $A$ and $B$ (solid lines) can be found in Table 1 . The dashed water retention curves are calculated from the values $\alpha(z)$ and $n(z)$ shown in Fig. 4.

Obviously, no unique hydraulic description can be obtained, since the results depend on the orientation of the sample. This is true despite a satisfactory performance of the inverse modeling procedure.

\section{Conclusions}

We demonstrated that the outflow from a weakly heterogeneous medium as measured in a multi-step outflow experiment can be described by assuming a uniform medium and an appropriate effective parameterization. However, x-ray measurements of $\theta(z)$ during the experiment revealed significant discrepancies between simulated and measured profiles. Allowing for a layered structure of the sample, these discrepancies could be reduced drastically without significantly affecting the simulated outflow. However, as we demonstrated numerically, such a model is not unique anymore since the effective properties now depend on the orientation of the sample.

We notice that the non-uniqueness cannot be identified with the traditional multi-step outflow experiment where flow is in one direction only. Even an additional imbibition experiment where the water is allowed to flow back into the column will not reveal it. Indeed, flow-back measurements always yield a set of parameters that differs from that of the outflow measurements. Up to now this is invariably ascribed to hysteresis, which as we demonstrated might not be the only reason for the deviation.

We comment that, by definition, the question of uniqueness does not arise if the sample is an REV, a representative elementary volume. However, we concede that the existence of an REV is difficult to ascertain for soils which typically exhibit a multi-scale heterogeneity and specifically for unsaturated flow in layered media.

As a remedy, we propose to change the standard procedure for determining effective properties such that the sample is measured in two directions. It may furthermore be worthwhile to use the resulting sets of parameters in numerical simulations to assess the uncertainty of the results.

Edited by: N. Romano

\section{References}

Arya, L. M. and Paris, J. F.: A physicoempirical model to predict the soil moisture characteristic from particle-size distribution and bulk density data, Soil Sci. Soc. Am. J., 45, 1023-1030, 1981.

Celia, M. A., Bouloutas, E. T., and Zarba, R. L.: A general massconservative numerical solution for the unsaturated flow equation, Water Resour. Res., 26, 1483-1496, 1990.

Hubbel, J. H.: Photon mass attenuation and energy-absorption coefficients from $1 \mathrm{keV}$ to $20 \mathrm{MeV}$, Int. J. Appl. Radiat. Isot., 33, 1269-1290, 1982.

Miller, E. E. and Miller, R. D.: Physical theory for capillary flow phenomena, J. Appl. Phys., 27, 324-332, 1956.

Mualem, Y.: A new model for predicting the hydraulic conductivity of unsaturated porous media, Water Resour. Res., 12, 513-522, 1976.

Press, W. H., Teukolsky, S. A., Vetterling, W. T., and Flannery, B. P.: Numerical recipes in C, Cambridge University Press, 1992.

Rajarama, H., Ferrand, L. A., and Celia, M. A.: Prediction of relative permeabilities for unconsolidated soils using pore-scale network models, Water Resour. Res., 33, 43-52, 1997.

van Dam, J. C., Stricker, J. N. M., and Droogers, P.: Inverse method to determine soil hydraulic functions from multistep outflow experiments, Soil Sci. Soc. Am. J., 58, 647-652, 1994.

van Genuchten, M. T.: A closed-form equation for predicting the hydraulic conductivity of unsaturated soils, Soil Sci. Soc. Am. J., 44, 892-898, 1980.

Vogel, H. J.: A numerical experiment on pore size, pore connectivity, water retention, permeability, and solute transport using network models, Europ. J. Soil Sci., 51, 99-105, 2000.

Zurmühl, T.: Capability of convection-dispersion transport models to predict transient water and solute movement in undisturbed soil columns, Journal of Contaminant Hydrology, 30, 101-128, 1998. 